\title{
Donor mitral valve repair in cardiac transplantation
}

\author{
Pedro E. Antunes, David Prieto, Luís Eugénio, and Manuel J. Antunes, Coimbra, Portugal
}

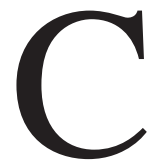

ardiac transplantation has become a relatively common procedure, but its major limiting factor is still the shortage of donor hearts. The donor pool can potentially be increased by relaxing selection criteria or by performing innovative procedures on the donor heart, such as valve repair. We present a recent case of successful transplantation after mitral valve repair in a heart with moderate rheumatic valve disease.

\section{Clinical Summary}

A 52-year-old man with end-stage congestive heart failure caused by idiopathic dilated cardiomyopathy was evaluated and listed for cardiac transplantation. On January 18, 2004, a 35year-old woman without a history of cardiac disease experienced brain death from a hemorrhagic cerebral accident and was selected as a multiorgan donor. The transthoracic echocardiogram showed evidence of mildly to moderately sclerotic leaflets with otherwise good motion and no evidence of stenosis (mitral valve area, $3.0 \mathrm{~cm}^{2}$ ) and mild mitral regurgitation. Regional and global myocardial contractility was unimpaired, and the ejection fraction was normal. Because there were no other contraindications to heart donation, we decided to proceed with the transplantation.

The heart was transported to our center, and the mitral valve was carefully inspected through the open left atrium. The free edges of the leaflets were mildly fibrosed and retracted, and the posteromedial commissure was moderately fused, as were the corresponding chordae tendineae. The valve was tested with injection of cold saline into the left ventricle after the aorta was clamped. There was a central jet of regurgitation caused by incomplete coaptation of the leaflets as a result of some retraction of the free edges. Because the valve disease was thought to be more significant than anticipated before the heart procurement, it was decided to proceed to bench repair of the mitral valve. A commissurotomy of the posteromedial commissure, followed by division of fused chordae tendineae, was performed. Additionally, although there was only mild annular enlargement, an annuloplasty was

\footnotetext{
From the Centre of Cardiothoracic Surgery, University Hospital, Coimbra, Portugal.

Received for publication March 19, 2004; accepted for publication April 6, 2004.

Address for reprints: Manuel J. Antunes, Centro de Cirurgia Cardiotorácica, Hospitais da Universidade, 3049 Coimbra Codex, Portugal (E-mail: antunes.cct.huc@mail.telepac.pt).

J Thorac Cardiovasc Surg 2005;129:227-8

$0022-5223 / \$ 30.00$

Copyright $(2) 2005$ by The American Association for Thoracic Surgery

doi:10.1016/j.jtcvs.2004.04.041
}

performed to increase the area of leaflet coaptation and to prevent future annular enlargement. The posterior annulus was plicated with a double continuous 3-0 polyester suture placed from trigone to trigone (Figure 1). The valve was again tested, and good coaptation of the leaflets was observed. This was a straightforward procedure with understandably excellent exposure, and it required less than 10 minutes of additional ischemic time.

The heart was them implanted by a bicaval anastomotic technique. The crossclamp time was 35 minutes. The ischemic time of the donor heart was 84 minutes. Intraoperative transesophageal echocardiography revealed minimal mitral regurgitation. Donor heart function was excellent. There was no transvalvular gradient, as determined by direct measurement of simultaneous left ventricular and left atrial pressures.

\section{Discussion}

Cardiac transplantation has become a relatively common procedure, with the major limiting factor being the shortage of donor hearts. A more immediate solution is to increase the yield of hearts from currently available donors. Originally based on the experience at Stanford, the criteria for donor selection have been expanded, and factors that made a heart unacceptable for transplantation are changing. In this context several recommendations have been made recently to expand the donor pool effectively without increasing the risk of adverse outcomes. ${ }^{1}$

One of these is related to valvular dysfunction, considering that in some cases bench repair can be performed on a donor heart with mild or moderate mitral or tricuspid regurgitation. Considering our own excellent results with mitral valve repair for mitral stenosis and annular dilatation, ${ }^{2}$ we believed we were justified to proceed with conventional mitral valve repair in this situation.

In the literature we could find reports of only 3 cases of mitral valve repair in valves without organic involvement in which the regurgitation was caused by annular dilatation., ${ }^{3,4}$ On the other hand, the presence of organic valve disease in a donor heart is usually considered an absolute contraindication to cardiac donation. To our knowledge, the only other case of repair of organic mitral valve disease (ie, open mitral commissurotomy) before orthotopic transplantation was reported in $1994 .^{5}$ The authors justified the decision mainly on the critical condition of the patient.

We report a case a mitral valve repair (commissurotomy plus annuloplasty) in a mildly dysfunctional valve caused by a rheumatic process. Our decision was made because we thought that the donor mitral valve could be made competent and the functional area could be slightly increased without detriment to the left ventricular function or compromise of the long-term durability of the organ. But this remains to be confirmed. However, we believe that immunosuppression therapy, especially steroids, can only slow the evolution of the scarring process. 


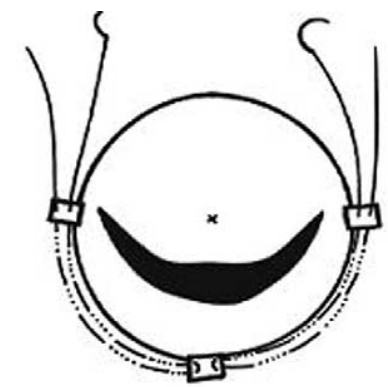

A

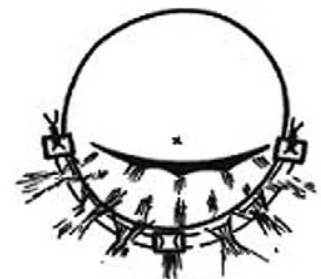

B
Figure 1. Technique of posterior annular plication used in this case.

\section{References}

1. Zaroff JG, Rosengard BR, Armstrong WF, Babcock WD, D'Alessandro A, Dec GW, et al. Consensus conference report: maximizing use of organs recovered from the cadaver donor: cardiac recommendations. Circulation. 2002;106:836-84.

2. Antunes MJ, Vieira H, Ferrão de Oliveira J. Open mitral commissurotomy: the "golden standard." J Heart Valve Dis. 2000;9:472-7.

3. Massad MG, Smedira NG, Hobbs RE, Hoercher K, Vandervoot P, McCarty PM. Bench repair of the donor mitral valve before heart transplantation. Ann Thorac Surg. 1996;61:1833-5.

4. Michler RE, Camacho DR. Ex-vivo mitral valve repair prior to orthotopic cardiac transplantation. Ann Thorac Surg. 2002;73:962-3.

5. Risher WH, Ochsner JL, Van Meter C. Cardiac transplantation after donor mitral valve commissurotomy. Ann Thorac Surg. 1994;57:221-2.

\section{Salmonella infection in an anterior mediastinal mass}

Yvonne M. Carter, MD, ${ }^{a}$ Lamia Shagrun, MD, ${ }^{b}$ Harvey Klein, MD, ${ }^{b}$ Jeffrey Katz, MD, ${ }^{c}$ and David M. Jablons, MD, ${ }^{a}$ San Francisco, Calif

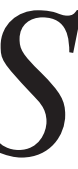

almonella infection occurs through fecal-oral transmission and usually appears in the biliary tract in the rare instance that it is extraintestinal. Thoracic Salmonella infection is rare $^{1-3}$ and has never been reported in the thymus. One case attributes a Salmonella thyroid abscess to the immunosuppressive therapy for myasthenia gravis. ${ }^{4}$ There has been only one previous report of thymic abscess in an adult in the literature. ${ }^{5}$ We report the first description of a Salmonella bacteremia associated with a thymic abscess within a thymoma.

\section{Clinical Summary}

Six years before presentation, a 55-year-old woman was found to have a large, anterior mediastinal mass on routine chest radiography. It was presumed to be a thymoma at that time, and she declined further diagnostic or therapeutic intervention.

At the time of presentation, the patient reported a 3-day history of fevers, polyarthralgias, rigors, chills, and pleuritic chest pain. She denied any symptoms suggestive of myasthenia gravis. Her

\footnotetext{
From the Division of Thoracic Surgery, ${ }^{\mathrm{a}}$ the Department of Pathology, ${ }^{\mathrm{b}}$ and the Department of Anesthesiology, ${ }^{\mathrm{c}}$ University of California, San Francisco Medical Center, San Francisco, Calif.

Received for publication March 18, 2004; accepted for publication April 6, 2004.

Address for reprints: David M. Jablons, MD, Thoracic Surgery, UCSF Medical Center, 505 Parnassus Ave, Box 0118, San Francisco, CA 94143 (E-mail: JablonsD@ @urgery.ucsf.edu).

J Thorac Cardiovasc Surg 2005;129:228-30

$0022-5223 / \$ 30.00$

Copyright $\odot 2005$ by The American Association for Thoracic Surgery doi:10.1016/j.jtcvs.2004.04.042
}

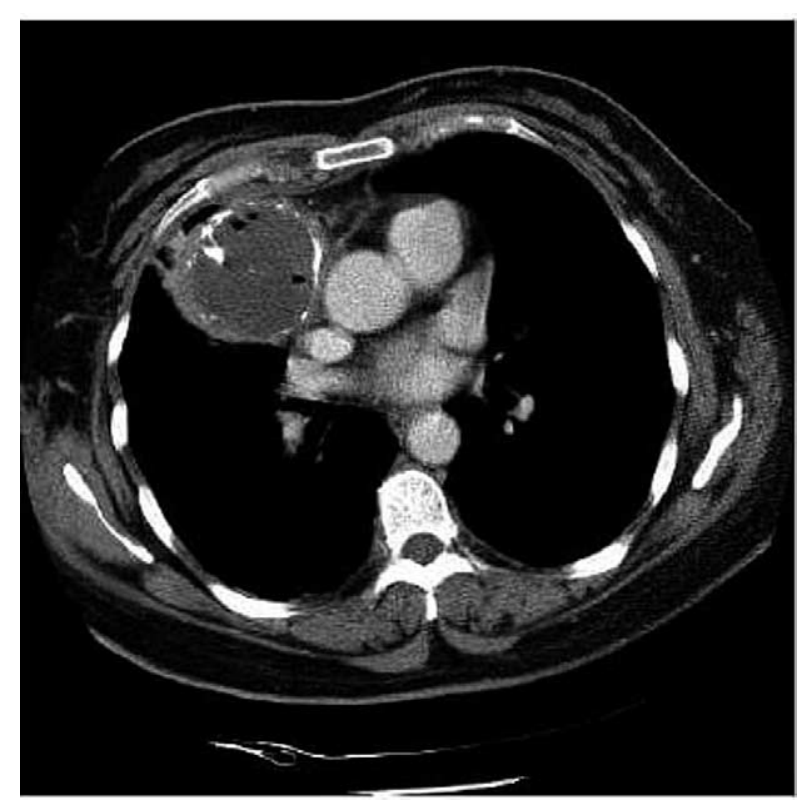

Figure 1. Chest computed tomographic scan of the $8 \times 10-\mathrm{cm}$ calcified mass in the anterior superior right mediastinum.

medical history included type II diabetes mellitus and leukocytosis and thrombocytosis of unknown cause.

Significant clinical findings on admission were a temperature of $101.7^{\circ} \mathrm{F}$ and a blood pressure of $90 / 50 \mathrm{~mm} \mathrm{Hg}$. Her white blood cell count was $24,600 / \mu \mathrm{L}$, with $66 \%$ polymorphonuclear cells and $17 \%$ bands. With prophylactic antibiotics, her white blood cell count decreased to $13,000 / \mu \mathrm{L}$; however, she continued to have 